\title{
High temperature carrier controlled ferromagnetism in alkali doped ZnO nanorods
}

\author{
Santa Chawla, ${ }^{\text {a) }}$ K. Jayanthi, and R. K. Kotnala \\ National Physical Laboratory, Council of Scientific and Industrial Research, Dr. K. S. Krishnan Road, New \\ Delhi 110012, India
}

(Received 6 August 2009; accepted 17 October 2009; published online 15 December 2009)

\begin{abstract}
Recent efforts in developing spintronic and magneto-optoelectric material for applications have relied on the use of magnetic semiconductors doped with transition metals and have met with limited success. Using a fresh synthesis approach using alkali ions we demonstrate that alkali doped zinc oxide can provide high temperature magnetic semiconductors. We report studies on nanocrystalline powder and pellets of $p$-type $\mathrm{ZnO}: \mathrm{Li}$ and $\mathrm{ZnO}: \mathrm{Na}$ that exhibit ferromagnetism up to $554 \mathrm{~K}$. The ferromagnetic behavior was confirmed from magnetic hysteresis, ferromagnetic resonance, magnetic force microscopy, and explained by a model where substitutional $\mathrm{Li}^{+} / \mathrm{Na}^{+}$in cation site induce local magnetic moments on oxygen atoms. Optimum dopant concentrations enable ferromagnetic exchange interaction leading to high Curie temperature. (C) 2009 American Institute of Physics. [doi:10.1063/1.3261722]
\end{abstract}

\section{INTRODUCTION}

Ideal materials and material properties for spintronics applications are: (1) dilute magnetic semiconductor (DMS) without any transition metal atoms, (2) independently controllable carrier density, and (3) carrier density dependent magnetization with high Curie temperature. We demonstrate that alkali atom $(\mathrm{Li} / \mathrm{Na})$ doped $\mathrm{ZnO}$ fulfills these conditions. Dietl et al. ${ }^{1}$ suggested that carrier induced ferromagnetism (FM) in Mn doped $p$-type material may be observed at higher temperature. Observation of carrier mediated FM in (Ga,Mn)As (Ref. 2) and the critical role of defects in DMS material in inducing FM resulted in numerous studies to achieve magnetic order at room temperatures (RTs) in oxide semiconductors. Observation of FM in wide band gap oxide materials (such as $\mathrm{HfO}_{2}, \mathrm{TiO}_{2}$, and $\mathrm{ZnO}$ ) arising either due to intrinsic vacancy defects ${ }^{3,4}$ or due to doping of $3 d$ transition metals ${ }^{5-9}$ has initiated new research. Dilute FM in $\mathrm{ZnO}$ with $3 d$ dopants (particularly with $\mathrm{Mn}, \mathrm{Co}, \mathrm{Ni}, \mathrm{Fe}, \mathrm{Cu}$, and $\mathrm{V})^{5-8}$ and carbon doping ${ }^{10}$ has been reported, however, concerns on the experimental results have been expressed. These relate to alternative possibilities on the origin of FM such as precipitation and clustering of doped magnetic atoms ${ }^{11-13}$ to even magnetic contamination during sample preparation. To avoid such possibilities, we examined the doping of monovalent alkali atom $\mathrm{Li}$ and $\mathrm{Na}$ with single unpaired spin in $\mathrm{ZnO}$. This is a case of FM in $p$-type $\mathrm{ZnO}$ without any transition metal. This is preferable for spintronic applications. ${ }^{14}$ The challenge of producing a DMS at RT was met by doping only hole in such a way that the interaction between holes localized spins on oxygen atoms and also mediated in long range ordering that is needed to sustain FM above RT. Such ordered magnetic moments induced by low concentration of monovalent alkali atom in $\mathrm{ZnO}$ are unprecedented and its explanation is a challenge for theoretical physics. As $\mathrm{ZnO}$ is

\footnotetext{
${ }^{a)}$ Author to whom correspondence should be addressed. Electronic mail:santa@nplindia.org.
}

a multifunctional semiconductor and emits UV and visible luminescence, ${ }^{5}$ RTFM in $\mathrm{ZnO}$ becomes promising for the development of devices based on spin transport properties such as spin-light emitting diode, ${ }^{5}$ optical isolators, optical switches, ${ }^{5}$ etc.

\section{EXPERIMENTAL PROCEDURES}

\section{A. Synthesis of $\mathrm{ZnO}: \mathrm{Li} / \mathrm{ZnO}: \mathrm{Na}$ nanorods}

Nanocrystalline powder of $\mathrm{ZnO}$ doped with $\mathrm{Li} / \mathrm{Na}$ was synthesized by solid state reaction method. High purity precursor materials $\mathrm{Zn}\left(\mathrm{CH}_{3} \mathrm{COO}\right)_{2} \cdot 2 \mathrm{H}_{2} \mathrm{O}$ and $\mathrm{LiOH} / \mathrm{NaOH}$ were homogenized in solution of de-ionized water and oven dried. Dried sample was ground, mixed well, and calcined in a quartz boat at $500{ }^{\circ} \mathrm{C}$ in air in a programmable furnace. The experiment was repeated for different $\mathrm{Li}$ and $\mathrm{Na}$ concentrations (2-15 at. \%). The synthesized $\mathrm{ZnO}: \mathrm{Li}$ and $\mathrm{ZnO}: \mathrm{Na}$ was grayish white powder. Trace magnetic impurities in the precursor materials were at parts-per-million level and the same precursor was used to synthesize undoped $\mathrm{ZnO}$. Any possibility of magnetic contamination through accidental or trace impurities were meticulously avoided during sample preparation and handling process. Only trace magnetic impurity present in the precursor material $\mathrm{Zn}\left(\mathrm{CH}_{3} \mathrm{COO}\right)_{2} \cdot 2 \mathrm{H}_{2} \mathrm{O}$, (99.5\%) was iron $0.0005 \%$ and in $\mathrm{LiOH} / \mathrm{NaOH} 0.005 \%$. Moreover, sample preparation was done in high purity quartz boat without any metallic contamination.

\section{B. Characterization}

Crystallographic phase identification was done by x-ray diffraction (XRD) (Brucker-AXS D8 Advance Diffractometer). Morphology was studied by transmission electron microscope (TEM) make JEM, model JEOL $200 \mathrm{Cx}$, and high resolution TEM (HRTEM). Energy dispersive x-ray spectroscopy (EDS) confirmed concentration of dopants. No trace magnetic impurity was detected in EDS measurements. Nature of electrical conduction was measured by Hall effect 
after pelletizing the powder. Pellets were made from $\mathrm{ZnO}: \mathrm{Li} /$ $\mathrm{ZnO}: \mathrm{Na}$ powder samples pressing them to 10 ton pressure. For resistivity and Hall effect measurements, Ohmic contacts were made on the pellets by silver paste followed by curing at $350{ }^{\circ} \mathrm{C}$ for $30 \mathrm{~min}$, followed by soldering the electrodes on silvered even surface. Hall effect measurement was done in Van der Pauw configuration using high resolution instruments (Keithley Source meter 6621, accuracy $\pm 0.1 \%$; Keithley Digital Voltmeter DVM 182, accuracy $\pm 0.05 \%$; Brucker Electromagnet system BE25, accuracy $\pm 1 \%$ ). Hall measurements were carried out even at different magnetic fields and different applied voltages on the same sample maintaining a constant current of $100 \mu \mathrm{A}$ and there was no appreciable change in measured hole concentration. The accuracy of Hall measurement was $\pm 1.5 \%$. Electron paramagnetic resonance (EPR) was performed on a Varian E-112 spectrometer at microwave frequency of $9.11 \mathrm{GHz}$ at ambient temperature. Magnetic measurements of $\mathrm{ZnO}: \mathrm{Li} / \mathrm{Na}$ powder samples were carried out using Lakeshore 7304 vibrating sample magnetometer (VSM). For magnetic measurement in VSM, the sample holder was of high purity perspex free from any metallic impurity. The holder was ultrasonically cleaned to remove any magnetic material traces and used exclusively for measurement of $\mathrm{ZnO}$ samples to avoid any magnetic contamination. Moreover, contribution from sample holder was subtracted from the sample signal. VSM has magnetization sensitivity $\sim 10^{-6} \mathrm{emu}$, while our experimental results show magnetization on the order of $10^{-3} \mathrm{emu}$. Temperature dependent magnetization study was carried out using VSM for Curie temperature determination. Magnetic measurements were repeatable for all the samples. $\mathrm{ZnO}: \mathrm{Li} / \mathrm{Na}$ samples with different $\mathrm{Li} / \mathrm{Na}$ concentrations were synthesized repeatedly in different batches under same experimental conditions and the results were consistent and reproducible. Magnetic force microscopy (MFM) was done with a high resolution scanning probe microscope with magnetic probe attachment for MFM analysis (SPM multimode V, Veeco Instruments Inc., USA).

\section{RESULTS AND DISCUSSION}

\section{A. XRD and HRTEM}

The synthesis of alkali doped $\mathrm{ZnO}$ was done by low temperature solid state reaction method starting from a uniform mixture of precursor components. This ensured homogenous doping as indicated by the shift in XRD peaks for undoped and doped $\mathrm{ZnO}$ ( $\mathrm{Li}$ and $\mathrm{Na}$ ), (Fig. 1). $\mathrm{ZnO}$ has a hexagonal wurtzite structure and the effect of doping was to increase the lattice parameters $a$ and $c$ by $<0.6 \%$. TEM images (Fig. 2) show well formed transparent nanorods of $\mathrm{ZnO}: \mathrm{Li}$ and $\mathrm{ZnO}: \mathrm{Na}$.

\section{B. Hall effect measurement}

Undoped $\mathrm{ZnO}$ exhibited $n$-type conductivity due to shallow donor levels. Hall effect measurement showed $p$-type conductivity in $\mathrm{ZnO}$ doped with $\mathrm{Li}$ and $\mathrm{Na}$. For $\mathrm{ZnO}: \mathrm{Li}$, hole concentration and resistivity varied from $1.6 \times 10^{16}$ to 1.3 $\times 10^{19} \mathrm{~cm}^{-3}$ and 107.5 to $43.7 \mathrm{k} \Omega \mathrm{cm}$, respectively, for variation in $\mathrm{Li}$ concentration from $2 \%$ to $15 \% .{ }^{15}$ The results

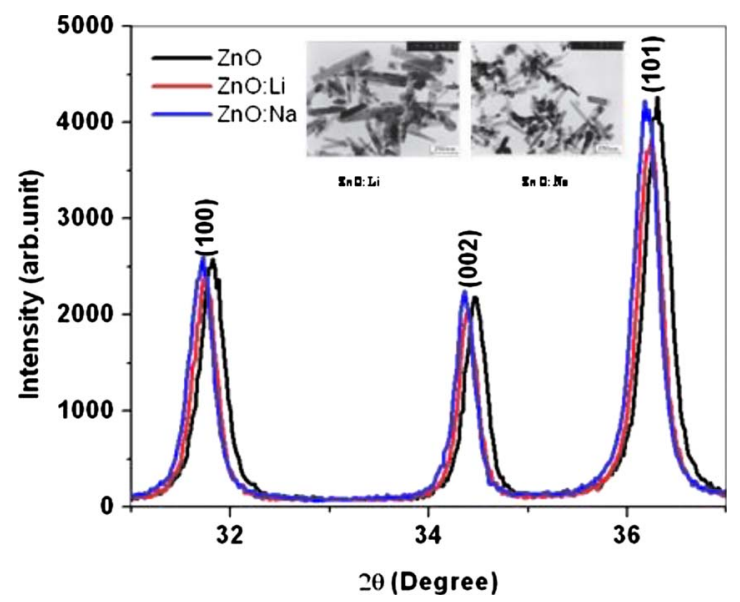

FIG. 1. (Color online) XRD spectra of undoped and Li, Na doped (2 at. \%) $\mathrm{ZnO}$ nanorods showing shift in the peaks toward lower angle due to doping. The inset shows the TEM images of corresponding $\mathrm{ZnO}: \mathrm{Li}$ and $\mathrm{ZnO}: \mathrm{Na}$ nanorods in same scale.

unambiguously indicate that the hole concentration increases and resistivity decreases with increase in $\mathrm{Li}$ concentration and accord with earlier reports ${ }^{16,17}$ on $\mathrm{Li}$ doped $\mathrm{ZnO}$ thin film deposited at $500{ }^{\circ} \mathrm{C}$. These indicated effective substitution of $\mathrm{Li}$ in zinc sites leading to $p$-type conductivity. ${ }^{16,17}$ The measured hole concentration was $7.5 \times 10^{16} \mathrm{~cm}^{-3}$ and resistivity $28.8 \mathrm{k} \Omega \mathrm{cm}$ for $\mathrm{ZnO}: \mathrm{Na}(10$ at \%). Though such measurements for $\mathrm{ZnO}: \mathrm{Na}$ are not available in literature, these values are similar to the values for $\mathrm{ZnO}: \mathrm{Li}$ synthesized at $500{ }^{\circ} \mathrm{C}$.

\section{Magnetic measurement}

A direct evidence of FM in alkali doped $\mathrm{ZnO}$ nanocrystalline powder was from precise measurement of sample magnetization using VSM. The hysteresis loops for the magnetization of $\mathrm{ZnO}: \mathrm{Li}$ and $\mathrm{ZnO}: \mathrm{Na}$ nanocrystals are shown in Fig. 3(a). Maximum saturation magnetization value observed was $55.71 \mathrm{memu} / \mathrm{g}$. The maximum magnetic moment was observed for 2 at. $\% \mathrm{Li}$ doped $\mathrm{ZnO}{ }^{15} \mathrm{ZnO}: \mathrm{Li}$ (2 at. \%) sample exhibited a hysteresis loop with Curie temperature as high as $554 \mathrm{~K}$. The hysteresis loop for $\mathrm{ZnO}: \mathrm{Na}(2$ at. \%) was narrower with decreased magnetic moment and Curie temperature compared to $\mathrm{ZnO}: \mathrm{Li}(2$ at. \%). These characteristics are typical of a ferromagnetic system. The magnetization curve for $\mathrm{ZnO}: \mathrm{Na}$ nanocrystals is shown in Fig. 3(b). The maximum magnetic moment of $40.92 \mathrm{memu} / \mathrm{g}$ was observed for 2 at. \% $\mathrm{Na}$ doped $\mathrm{ZnO}$ which exhibited a typical ferromagnetic narrow hysteresis loop with Curie temperature of 396 K. FM declined with further increase in $\mathrm{Li} / \mathrm{Na}$ concentration in $\mathrm{ZnO}$ lattice and 15 at. \% Li/Na doped $\mathrm{ZnO}$ showed diamagnetism. Undoped $\mathrm{ZnO}$ synthesized in exactly identical experimental conditions exhibited diamagnetism. $\mathrm{ZnO}$ doped with potassium $(\mathrm{K})$ also showed diamagnetism. Table I collates the magnetization data for alkali doped $\mathrm{ZnO}$ nanocrystals. As zinc acetate is the common precursor for preparation of $\mathrm{ZnO}, \mathrm{ZnO}: \mathrm{Li}$, and $\mathrm{ZnO}: \mathrm{Na}$ system, its effect in producing FM can be ruled out immediately as undoped $\mathrm{ZnO}$ show pure diamagnetism. For 2 at. \% Li/ $\mathrm{Na}$ doped $\mathrm{ZnO}$, magnetization is maximum and trace iron $(\mathrm{Fe})$ would be only 

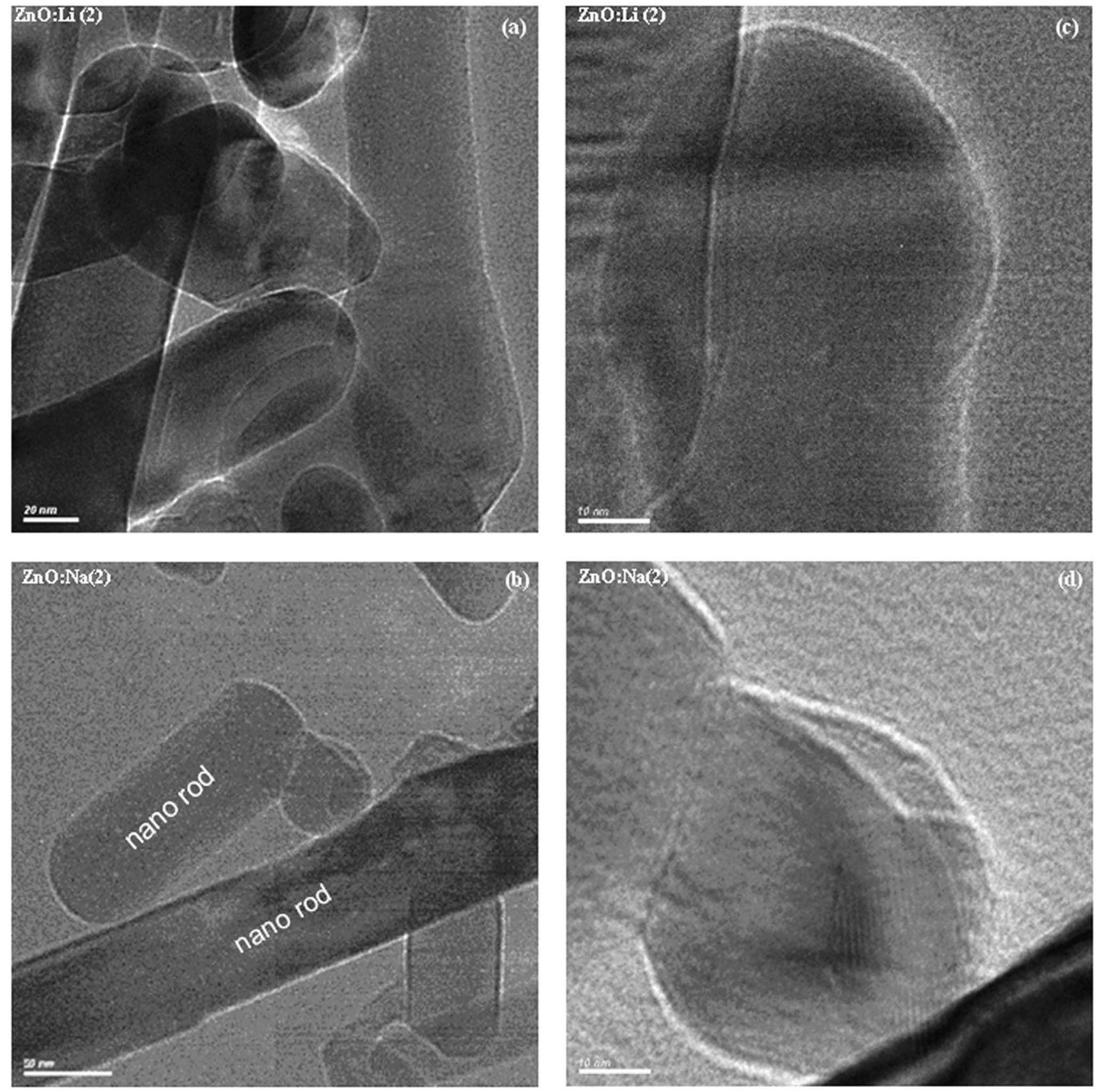

FIG. 2. High resolution TEM images of $\mathrm{ZnO}: \mathrm{Li}(2$ at. \%) and $\mathrm{ZnO}: \mathrm{Na}(2$ at. \%) showing well-formed nanorods and lattice fringes.

$0.0001 \%$ and as $\mathrm{Li} / \mathrm{Na}$ concentration increased, the amount of trace Fe should increase proportionately. But all the magnetic measurement results conclusively show that FM decreases with increase in $\mathrm{Li} / \mathrm{Na}$ concentration.

The measured saturation magnetization values are significantly higher than the reported magnetization values of non magnetic oxides ${ }^{3,4}$ and carbon doped $\mathrm{ZnO}$ films prepared by pulsed laser deposition technique. ${ }^{10}$ In $p$-type $\mathrm{ZnO}: \mathrm{Li}$ (Ref. 15) and ZnO:Na systems, exhibiting RTFM, the saturation magnetization is intricately interlinked with carrier density as magnetization decreases with increase in hole concentration, and a cause-effect relationship with hole doping and ferromagnetic ordering is inescapable. Having been synthesized using identical procedures, the only difference between diamagnetic $\mathrm{ZnO}$ and ferromagnetic $\mathrm{ZnO}: \mathrm{Li}$ and
$\mathrm{ZnO}: \mathrm{Na}$ is the presence of $\mathrm{Li} / \mathrm{Na}$ atom at zinc sites. These produce unpaired spin and consequently the origin of FM must relate to these defects.

\section{Ferromagnetic resonance}

Detection of ferromagnetic ordering by ferromagnetic resonance (FMR) is one of the most sensitive methods capable of detecting as low as $10^{13}$ spins along with other magnetic species. FM in $\mathrm{ZnO}: \mathrm{Li}$ and $\mathrm{ZnO}: \mathrm{Na}$ powder samples was clearly manifested by a single resonance (line width 3.5 Gauss) in EPR spectra (Fig. 4) observed at RT. The signal intensity in $\mathrm{ZnO}: \mathrm{Na}$ system is lower than the value observed in $\mathrm{ZnO}: \mathrm{Li}$ system. Broad FMR arises due to transition within ground state of a ferromagnetic domain. As the 


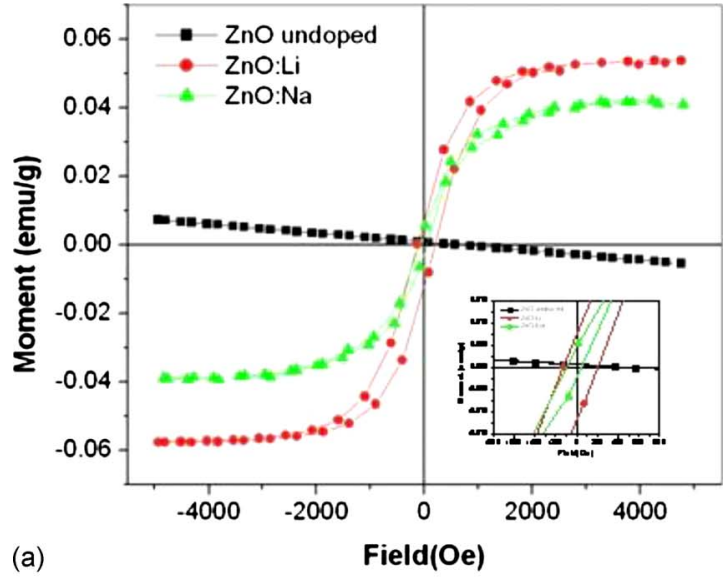

ZnO:Na

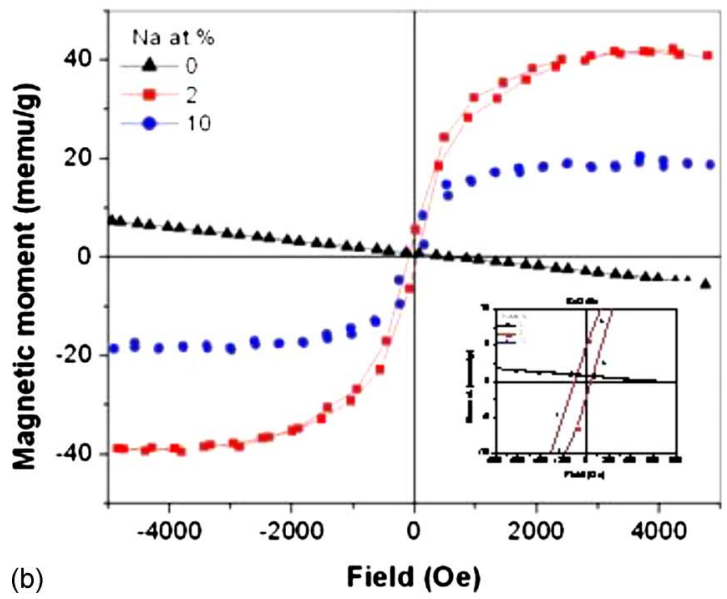

FIG. 3. (Color online) (a) Magnetization curve of Li/Na (2 at. \%) doped $\mathrm{ZnO}$ nanocrystals showing typical ferromagnetic hysteresis loop with maximum magnetization for 2 at. \% $\mathrm{Li}$ doping in $\mathrm{ZnO}$. Undoped $\mathrm{ZnO}$ exhibits diamagnetism. The inset shows magnified view to show coercive field and remanence. (b) Magnetization curve of $\mathrm{ZnO}$ : Na nanocrystals showing typical ferromagnetic hysteresis loop with maximum magnetization for 2 at. \% $\mathrm{Na}$ doping in $\mathrm{ZnO}$. Undoped $\mathrm{ZnO}$ exhibits diamagnetism. The inset shows magnified view to show coercive field and remanence.

measurements were on nanocrystalline powder, the broadness of the spectra could arise due to intrinsic anisotropy, orientation and randomness of powder samples, multiplicity of the ferromagnetic domain's ground state, and inhomogeneous domain size. Presence of such a FMR at RT and the absence of typical Li/Na EPR structure ${ }^{18,19}$ attests to the presence of ferromagnetic phase in ZnO:Li (Ref. 20) and $\mathrm{ZnO}: \mathrm{Na}$ nanocrystalline powder samples. Such broad FMR spectra has also been reported for $\mathrm{Mn}^{2+}$ doped $\mathrm{ZnO}$ pellets sintered at $500{ }^{\circ} \mathrm{C}^{8} \mathrm{Mn}^{2+}$ doped $\mathrm{ZnO}$ quantum dots, ${ }^{21}$ $\mathrm{Ni} / \mathrm{ZnO},{ }^{22}$ and $(\mathrm{Ga}, \mathrm{Mn}) \mathrm{As}^{23}$

TABLE I. Curie temperature and saturation magnetic moment for $\mathrm{ZnO}$ doped with $\mathrm{Li}, \mathrm{Na}$, and $\mathrm{K}$.

\begin{tabular}{lcc}
\hline \hline $\begin{array}{l}\text { Dopant } \\
\text { (at. \%) }\end{array}$ & $\begin{array}{c}T_{C} \\
(\mathrm{~K})\end{array}$ & $\begin{array}{c}\text { Maximum magnetic moment } \\
(\mathrm{memu} / \mathrm{g})\end{array}$ \\
\hline $\mathrm{Li}(2)$ & 554 & 55.71 \\
$\mathrm{Na}(2)$ & 396 & 40.92 \\
$\mathrm{~K}(2)$ & diamagnetic & diamagnetic \\
\hline \hline
\end{tabular}

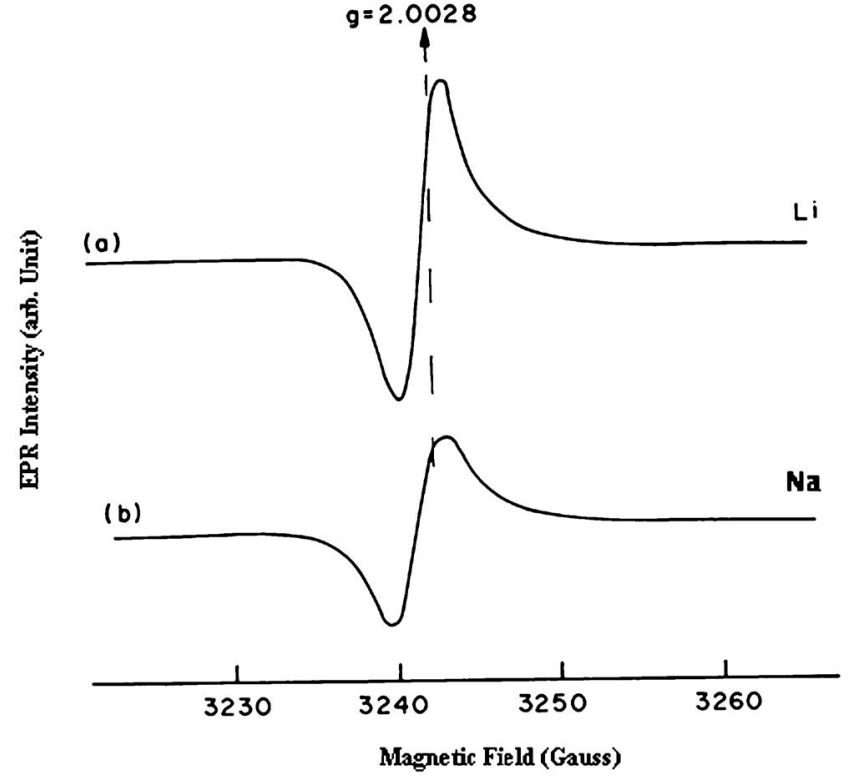

FIG. 4. FMR spectra of $\mathrm{ZnO}: \mathrm{Li}$ and $\mathrm{ZnO}: \mathrm{Na}$ powder sample at RT. The vertical line shows $g$ value of tetracyanoethylene (TCNE, $g=2.0028)$ which was used as a standard. The single line observed at RT corresponds to FMR.

\section{E. MFM}

Formation of magnetic domains is basic to the origin of $\mathrm{FM}$, hence the magnetic microstructure was investigated by high resolution MFM on pellets of $\mathrm{ZnO}: \mathrm{Li}$ and $\mathrm{ZnO}: \mathrm{Na}$ nanocrystals.

The magnetic domains and their variation with $\mathrm{Li} / \mathrm{Na}$ concentration is shown in Fig. 5 in a $1 \times 1 \mu \mathrm{m}^{2}$ area image. It clearly shows that $\mathrm{ZnO}: \mathrm{Li}(2$ at. \%) exhibited magnetic domains with a near uniform distribution at RT and the pattern changes with increase in dopant concentration. The domain pattern and its variation with $\mathrm{Li} / \mathrm{Na}$ concentration became apparent in Figs. 5(a)-5(d). For 2 at. \% Li doped $\mathrm{ZnO}$, the magnetic domains [Fig. 5(a)] are isolated and oriented producing maximum magnetization as is also manifested in $M-H$ loop [Fig. 3(a)]. With an increase in Li concentration, the domains [Fig. 5(b)] become clustered, less oriented, and nonuniformly distributed thereby decreasing the overall magnetization. ${ }^{20}$ For $\mathrm{ZnO}: \mathrm{Na}$ (2 at. \%) [Fig. 5(c)], the magnetic domains are distributed along curved lines forming open ended pattern. In 10 at. \% $\mathrm{Na}$ doped $\mathrm{ZnO}$, nonoriented domains [Fig. 5(d)] are observed. Magnetic domain structure clearly visible in the MFM images thus support the existence of FM and its variation with $\mathrm{Li} / \mathrm{Na}$ concentration in $\mathrm{ZnO}$. Uniform and oriented domains for $\mathrm{ZnO}: \mathrm{Li}(2$ at. \%) give rise to maximum magnetic moment and $T_{C}$. The orientation and distribution of domains become nonuniform as $\mathrm{Li}$ concentration increases as well as overlapping of domains occurs resulting in decrease in magnetization. Such a domain mapping by MFM provides a conclusive evidence of FM, As this has been reported for many DMS, ${ }^{9,24,25}$ it is inferred that observed $\mathrm{FM}$ in $\mathrm{Li}$ and $\mathrm{Na}$ doped $\mathrm{ZnO}$ is an intrinsic property of the material arising due to alkali doping.

\section{F. Theoretical explanation}

Cationic substitutional defects $(\mathrm{Li} / \mathrm{Na})$ can induce local magnetic moments on neighboring oxygen atoms and Bou- 

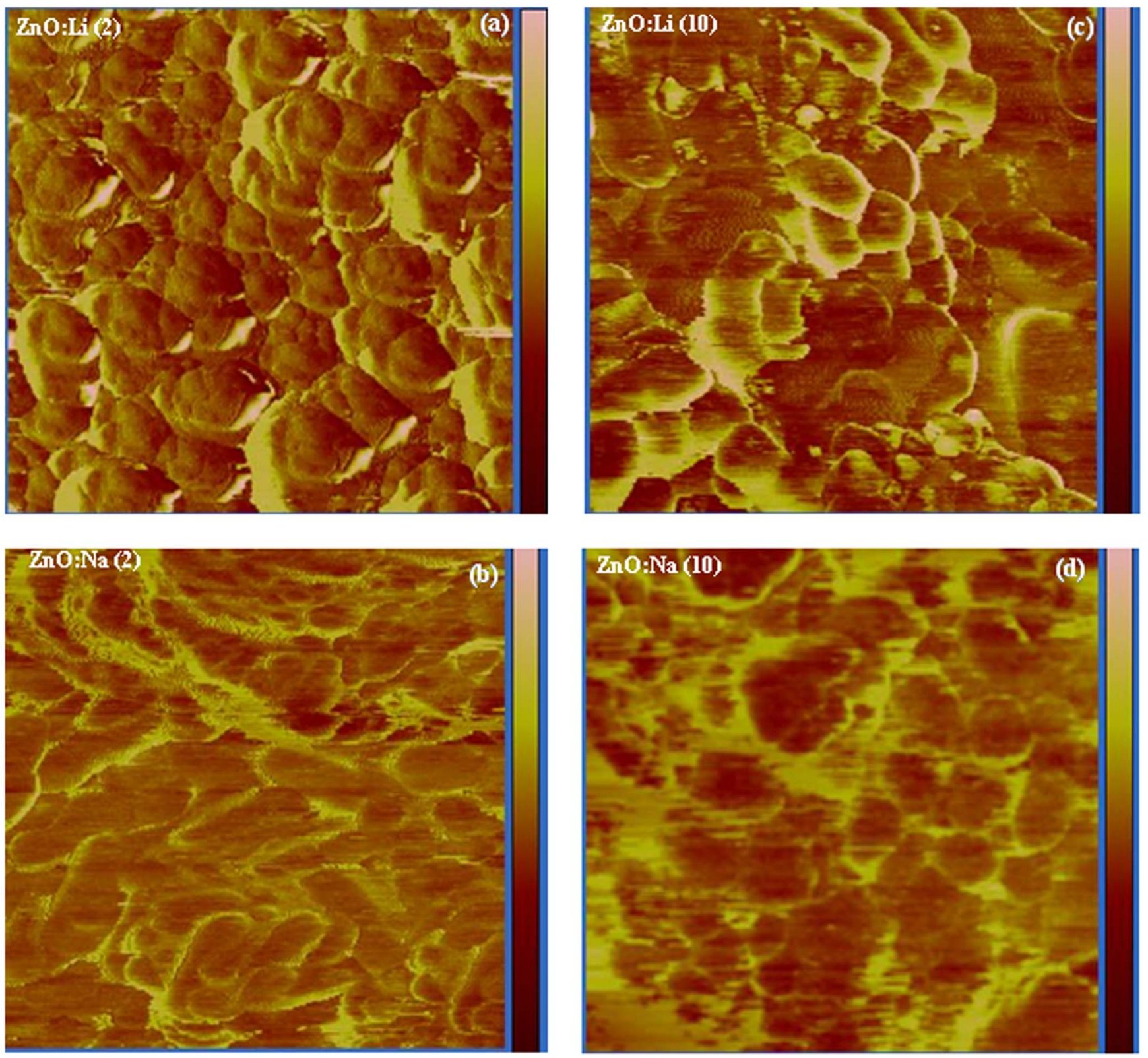

FIG. 5. (Color online) MFM picture of pellet made of $\mathrm{ZnO}: \mathrm{Li}$ and $\mathrm{ZnO}$ :Na nanorods in a $1 \times 1 \mu \mathrm{m}^{2}$ area image, the domain structure and its variation with $\mathrm{Li} / \mathrm{Na}$ concentration is apparent. The number within bracket indicates the concentration of dopant in atomic percent. In the right hand scale, the variation in magnetic force with doping concentration is also apparent from the color scale with pink indicating maximum force.

zerar et al. ${ }^{20}$ suggested that such magnetic moments can produce long range ferromagnetic order and result in Curie temperature above RT. In theories of DMS, substitutional magnetic impurity creates localized magnetic moments which interact through carrier mediation. The subtle difference of such theory with the present case is that localized moments are induced on nonmagnetic oxygen atoms and distinction between holes localizing the moments and mediating the long range order is not apparent. The effect of cationic substitutional defects $(\mathrm{Li} / \mathrm{Na})$ is taken as additional attractive, locally correlated random potential acting on a single correlated band of oxygen orbitals. Shallow acceptor level produced by $\mathrm{Li} / \mathrm{Na}$ dopant introduces holes and hole density is taken as a variable. For certain hole density, moments start appearing and can get saturated at optimum values of defect concentration. The magnetic coupling critically depends on the value of the potential. For small as well as high values of potential, the nearest neighbor couplings are antiferromagnetic and these fluctuate between ferromagnetic and antiferromagnetic with distance. In the model proposed by Bouzerar et al., ${ }^{20}$ for potential values in the range of approximately $35 \%-55 \%$ of the host bandwidth, the coupling is ferromagnetic which leads to ferromagnetic exchange interaction and enables a higher (above RT) Curie temperature $\left(T_{C}\right)$. For values of potential outside the particular range, $T_{C}$ vanishes as for smaller potential values Ruderman-Kittel-Kasya-Yashida type oscillations or for higher potential values superexchange destroys the coupling. ${ }^{20}$ The value of potential where strong ferromagnetic coupling and above RT $T_{C}$ is predicted also show formation of well-defined impurity band split from the valence band edge in the density of states. Such a behavior has also 
been reported for $\mathrm{Ga}(\mathrm{Mn}) \mathrm{As}$ (Ref. 26) and $\mathrm{CaO}^{27}$ The concentration of defects play an important role in terms of carrier density and higher Curie temperature (above RT) is possible only for a limited range of dopant concentration, i.e., carrier densities. The optimum hole density to maintain long range ferromagnetic order for the $\mathrm{Li} / \mathrm{Na}$ doped $\mathrm{ZnO}$ system is $\sim 10^{16}-10^{18} \mathrm{~cm}^{-3}$ (Table I). Lower hole density $\left(\sim 10^{16} \mathrm{~cm}^{-3}\right)$ produces maximum ferromagnetic coupling and Curie temperature $(554 \mathrm{~K})$. With increase in $\mathrm{Li} / \mathrm{Na}$ concentration the resulting potential and hole density would destroy long range ferromagnetic order whereas local moments would still exist. We measured RTFM in Li/Na concentration range of 2-10 at. \%. These introduced varying amount of hole density and influenced variation in extent of magnetization and Curie temperature. FM has not been observed for dopant concentration more than 10 at. \%. The present work thus leads to experimental proof of the theory by Bouzerer $e t$ $a l^{20}$ A quantitative model to explain the observed effects, however, is awaited. Also, the atomic number of dopant influences the value of the potential and ferromagnetic exchange interaction since magnetization declined from $\mathrm{Li}$ to $\mathrm{Na}$ and not observed at all for $\mathrm{K}$ doped $\mathrm{ZnO}$. The stabilization of high temperature $T_{C}$ by substitution of nonmagnetic dopants such as $\mathrm{Li}$ and $\mathrm{Na}$ clearly indicate the definitive role of such spin $1 / 2$, hole generating dopants in creating long range ferromagnetic ordering in $\mathrm{ZnO}$ in certain doping range.

\section{CONCLUSIONS}

The present study established that nanocrystalline powder and pellets of $\mathrm{ZnO}: \mathrm{Li}$ and $\mathrm{ZnO}: \mathrm{Na}$ exhibit ferromagnetic order up to $554 \mathrm{~K}$. Such a unique ferromagnetic property of $\mathrm{Li} / \mathrm{Na}$ doped $\mathrm{ZnO}$ is due to low temperature synthesis process developed by us which induces shallow acceptor levels formed by $\mathrm{Li} / \mathrm{Na}$ in zinc substitutional sites. This procedure eliminates all the possibilities of contamination by magnetic elements and enables the conclusion that observed RTFM is an intrinsic property of $\mathrm{ZnO}: \mathrm{Li}$ and $\mathrm{ZnO}: \mathrm{Na}$ nanocrystals. Carrier density dependence on saturation magnetization and high Curie temperature of 344-554 K has been established with measurement of hole density and magnetic properties on same samples. FM at RT has been unequivocally supported with formation of clear magnetic domains and FMR in addition to narrow $M-H$ hysteresis loop. Substitutional $\mathrm{Li}^{+} / \mathrm{Na}^{+}$dopant induce moments on neighboring oxygen atoms which when considered in a correlated model for oxygen orbitals with random potentials may explain the observed FM and high Curie temperature.

Alkali ion doping induces intrinsic $\mathrm{FM}$ in $\mathrm{ZnO}$. Thus alkali doped $\mathrm{ZnO}$ could prove to be a better DMS material compared to transition metal ions where origin of FM due to precipitation/clustering of magnetic dopants always cast a doubt. Such novel DMS based on a multifunctional material such as $\mathrm{ZnO}$ could pave the way for a new class of magnetooptic and spintronic material for variety of applications. As synthesized ferromagnetic $p$-type $\mathrm{ZnO}: \mathrm{Li} / \mathrm{Na}$ emits strong near UV light, a distinct possibility of fabricating spin light emitting diode using such material could open up. Since the magnetic and spin dopants are the same element, spinpolarized current can also be a reality in such material.

\section{ACKNOWLEDGMENTS}

We wish to thank Dr. T. K. Gundu Rao for EPR, Dr. Sukhvir Singh for TEM, Ms. Jyoti Shah for Hall effect, Mr. S. B. Samanta for MFM measurements, and Professor A. K. Singhvi for critical assessment of the manuscript.

${ }^{1}$ T. Dietl, H. Ohno, J. Cibert, and D. Ferrand, Science 287, 1019 (2000).

${ }^{2}$ S. J. Potashnik, K. C. Ku, S. H. Chun, J. J. Berry, N. Samarth, and P. Schiffer, Appl. Phys. Lett. 79, 1495 (2001).

${ }^{3}$ M. Venkatesan, C. B. Fitzgerald, and J. M. D. Coey, Nature (London) 430, 630 (2004).

${ }^{4}$ A. Sundareshan R. Bhargavi, N. Rangarajan, U. Siddesh, and C. N. R. Rao, Phys. Rev. B 74, 161306 (2006).

${ }^{5}$ U. Ozgur, U. Ozgur, Ya. I. Alivov, C. Liu, A. Teke, M. A. Reshchikov, S. Dogan, V. Avrutin, S.-J. Cho, and H. Morkoc, J. Appl. Phys. 98, 041301 (2005) and references therein; I. A. Buyonova et al., J. Vac, Sci. Technol. B 24, 259 (2006); S. A. Chambers, Mater. Today 5, 34 (2002); H. Ohno et al., JSAP Int. 5, 4 (2002).

${ }^{6}$ S. J. Pearton, C. R. Abernathy, M. E. Overberg, G. T. Thaler, D. P. Norton, N. Theodoropoulou, A. F. Hebard, Y. D. Park, F. Ren, J. Kim, and L. A. Boatner, J. Appl. Phys. 93, 1 (2003).

${ }^{7}$ J. M. D. Coey, M. Venkatesan, and C. B. Fitzgerald, Nature Mater. 4, 173 (2005).

${ }^{8}$ P. Sharma, A. Gupta, K. V. Rao, F. J. Owens, R. Sharma, R. Ahuja, J. M. O. Guillen, B. Johansson, and G. A. Gehring, Nature (London) 2, 673 (2003).

${ }^{9}$ J. Philip, A. Punnoose, B. I. Kim, K. M. Reddy, S. Layne, J. O. Holmes, B. Satpati, P. R. LeClair, and T. S. Santos, Nature Mater. 5, 298 (2006).

${ }^{10}$ H. Pan, J. B. Yi, L. Shen, R. Q. Wu, J. H. Yang, J. Y. Lin, Y. P. Feng, J. Ding, L. H. Van, and J. H. Yin, Phys. Rev. Lett. 99, 127201 (2007).

${ }^{11}$ P. A. Stampe, R. J. Kennedy, Y. Xin, and J. S. Parker, J. Appl. Phys. 92, 7114 (2002).

${ }^{12}$ S. A. Chambers, T. Droubay, C. M. Wang, A. S. Lea, R. F. C. Farrow, L. Folks, V. Deline, and S. Anders, Appl. Phys. Lett. 82, 1257 (2003).

${ }^{13}$ J. H. Kim, H. Kim, D. Kim, Y. E. Ihm, and W. K. Choo, J. Appl. Phys. 92 , 6066 (2002).

${ }^{14}$ M. H. F. Sluiter, Y. Kawazoe, P. Sharma, A. Inoue, A. R. Raju, C. Rout, and U. V. Waghmare, Phys. Rev. Lett. 94, 187204 (2005).

${ }^{15}$ S. Chawla, K. Jayanthi, and R. Kotnala, Phys. Rev. B 79, 125204 (2009).

${ }^{16}$ Y. J. Zeng, Appl. Phys. Lett. 88, 062107 (2006).

${ }^{17}$ X. H. Wang, Z. Z. Ye, W. Z. Xu, D. Y. Li, J. G. Lu, L. P. Zhu, and B. H. Zhao, J. Phys. D: Appl. Phys. 39, 4568 (2006).

${ }^{18}$ J. Sann, A. Hofstaetter, D. Pfisterer, J. Stehr, and B. K. Meyer, Phys. Status Solidi C 3, 952 (2006).

${ }^{19}$ B. K. Meyer, A. Hofstaetter, and V. V. Laguta, Physica B 376-377, 682 (2006).

${ }^{20}$ G. Bouzerar and T. Ziman, Phys. Rev. Lett. 96, 207602 (2006).

${ }^{21}$ N. S. Norberg, K. R. Kittilstved, J. E. Amonette, R. K. Kukkadapu, D. A. Schwartz, and D. R. Gamelin, J. Am. Chem. Soc. 126, 9387 (2004).

${ }^{22}$ V. Castel, J. B. Youssef, and C. Brosseau, J. Nanomater., Special Issue 1, 2007 (2007).

${ }^{23}$ M. Rubinstein, A. Hanbicki, P. Lubitz, M. Osofsky, J. J. Krebs, and B. Jonker, J. Magn. Magn. Mater. 250, 164 (2002).

${ }^{24}$ H. C. Jeon, Y. S. Jeong, T. W. Kang, T. W. Kim, K. J. Chung, K. J. Chung, W. Jhe, and S. A. Song, Adv. Mater. 14, 1725 (2002).

${ }^{25}$ M. Diaconu, H. Schmidt, H. Hochmuth, M. Lorenz, G. Benndorf, J. Lenzner, D. Spemann, A. Setzer, K.-W. Nielsen, P. Esquinazi, and M. Grundmann, Thin Solid Films 486, 117 (2005).

${ }^{26}$ J. Kudrnovsky, I. Turek, V. Drchal, F. Maca, P. Weinberger, and P. Bruno, Phys. Rev. B 69, 115208 (2004).

${ }^{27}$ I. S. Elfimov, S. Yunoki, and G. A. Sawatzky, Phys. Rev. Lett. 89, 216403 (2002). 